\title{
N-Terminal Pro-Brain Natriuretic Peptide and Cognitive Decline in Older Adults at High Cardiovascular Risk
}

\author{
Liselotte W. Wijsman, MD, ${ }^{1,2}$ Behnam Sabayan, MD, MSc, PhD, ${ }^{1,3}$ \\ Peter van Vliet, MD, PhD, ${ }^{1,4}$ Stella Trompet, $\mathrm{PhD},{ }^{1,5}$ \\ Wouter de Ruijter, MD, PhD, ${ }^{6}$ Rosalinde K. E. Poortvliet, MD, ${ }^{6}$ \\ Petra G. van Peet, MD, ${ }^{6}$ Jacobijn Gussekloo, MD, PhD, ${ }^{6}$ \\ J. Wouter Jukema, MD, PhD, ${ }^{5}$ David J. Stott, MD, PhD, ${ }^{7}$ \\ Naveed Sattar, MD, PhD, ${ }^{8}$ lan Ford, PhD, ${ }^{9}$ Rudi G. J. Westendorp, MD, PhD, ${ }^{10}$ \\ Anton J. M. de Craen, PhD, ${ }^{1,2}$ and Simon P. Mooijaart, MD, PhD ${ }^{1,2,11}$
}

Objective: Elevated levels of N-terminal pro-brain natriuretic peptide (NT-proBNP) are associated with cognitive impairment, which might be explained by cardiovascular diseases or risk factors. The aim of this study was to investigate the association of NT-proBNP with cognitive function and decline in older adults at high risk of cardiovascular disease.

Methods: We studied 5,205 men and women (mean age $=75$ years) who were recruited into the PROspective Study of Pravastatin in the Elderly at Risk. All participants had pre-existing cardiovascular disease or risk factors thereof. Four domains of cognitive function were tested at baseline and repeated during a follow-up period of 3.2 years.

Results: Participants with higher NT-proBNP $(\geq 450 \mathrm{ng} / \mathrm{l})$ had worse baseline cognitive function, including reaction time (mean difference high vs low group $=3.07$ seconds, $95 \%$ confidence interval $[\mathrm{Cl}]=0.83$ to 5.32 ), processing speed ( -1.02 digits coded, $95 \% \mathrm{Cl}=-1.65$ to -0.39 ), and immediate memory $(-0.13$ pictures remembered, $95 \%$ $\mathrm{Cl}=-0.29$ to 0.04$)$. There was no significant difference in delayed memory $(-0.14,95 \% \mathrm{Cl}=-0.38$ to 0.10$)$ between the NT-proBNP groups. Participants with higher NT-proBNP had a steeper cognitive decline, including reaction time (mean annual change high vs low group $=0.60$ seconds, $95 \% \mathrm{Cl}=0.14$ to 1.07 ), processing speed ( -0.15 digits coded, $95 \% \mathrm{Cl}=-0.25$ to -0.05 ), immediate memory $(-0.05$ pictures remembered, $95 \% \mathrm{Cl}=-0.09$ to 0.00 ), and delayed memory $(-0.05$ pictures remembered, $95 \% \mathrm{Cl}=-0.11$ to 0.01$)$. Associations were independent of cardiovascular diseases and risks.

Interpretation: Higher NT-proBNP associates with worse cognitive function and steeper cognitive decline, independent of cardiovascular diseases and risks. Further studies to unravel the underlying mechanisms are warranted.

ANN NEUROL 2014;76:213-222

$\mathrm{H}$ igher levels of $\mathrm{N}$-terminal pro-brain natriuretic peptide (NT-proBNP), a neurohormone produced by cardiomyocytes in response to ventricular stretch, have been associated with cognitive impairment. ${ }^{1-4}$ Evidence comes from several cross-sectional studies, which show that among community-dwelling older adults, higher NT-proBNP levels were associated with worse cognitive function, in particular memory. ${ }^{1-4}$ There are limited

View this article online at wileyonlinelibrary.com. DOI: 10.1002/ana.24203

Received Feb 14, 2014, and in revised form Jun 16, 2014. Accepted for publication Jun 16, 2014.

Address correspondence to Dr Mooijaart, Department of Gerontology and Geriatrics, Leiden University Medical Center, PO Box 9600,2300 RC Leiden, the Netherlands. E-mail: s.p.mooijaart@lumc.nl

From the ${ }^{1}$ Department of Gerontology and Geriatrics, Leiden University Medical Center, Leiden, the Netherlands; ${ }^{2}$ Netherlands Consortium for Healthy Ageing, Leiden, the Netherlands; ${ }^{3}$ Department of Radiology, Leiden University Medical Center, Leiden, the Netherlands; ${ }^{4}$ Department of Neurology, Leiden University Medical Center, Leiden, the Netherlands; ${ }^{5}$ Department of Cardiology, Leiden University Medical Center, Leiden, the Netherlands; ${ }^{6}$ Department of Public Health and Primary Care, Leiden University Medical Center, Leiden, the Netherlands; ${ }^{7}$ Academic Section of Geriatric Medicine, Faculty of Medicine, University of Glasgow, Glasgow, United Kingdom; ${ }^{8}$ British Heart Foundation, Glasgow Cardiovascular Research Centre, University of Glasgow, Glasgow, United Kingdom; ${ }^{9}$ Robertson Center for Biostatistics, University of Glasgow, Glasgow, United Kingdom; ${ }^{10}$ Leyden Academy on Vitality and Ageing, Leiden, the Netherlands; and ${ }^{11}$ Institute for Evidence-Based Medicine in Old Age, Leiden, the Netherlands. 
numbers of longitudinal studies with relatively small sample sizes, which demonstrate that higher NT-proBNP levels are also associated with steeper declines in MiniMental State Examination (MMSE) scores and higher incidence of dementia. ${ }^{5,6}$ A potential mechanism behind the relationship between higher NT-proBNP levels and cognitive function is clinical heart failure, resulting in left ventricular dysfunction with subsequent reduced cardiac output. It is hypothesized that reduced cardiac output causes inadequate cerebral perfusion, leading to a higher risk of cognitive impairment. ${ }^{7-9}$ Improvements in cognitive function in patients following cardiac transplantation suggest that impaired cardiac function might be a reversible risk factor for cognitive impairment. ${ }^{10,11}$

Recent evidence demonstrates that higher NTproBNP levels in older adults are strongly associated with cardiovascular diseases and risk factors and predict an increased risk of atrial fibrillation, stroke, transient ischemic attack (TIA), myocardial infarction, and mortality, even in the absence of clinical heart failure. ${ }^{12-15}$ In addition, higher NT-proBNP levels have been related to left ventricular hypertrophy and systolic and diastolic dysfunction in adults without clinical heart failure. ${ }^{16,17}$ The relationship of cardiovascular diseases and risk factors with cognitive impairment is well established. ${ }^{18,19}$ Hence, cognitive impairment might already be present in asymptomatic older adults at early stages of reduced cardiac function.

We hypothesized that elevated levels of NTproBNP are associated with a steeper cognitive decline in older adults, which might be explained by cardiovascular diseases or risk factors. Therefore, we studied the crosssectional and longitudinal association of NT-proBNP with cognitive function in a cohort of older men and women from the PROspective Study of Pravastatin in the Elderly at Risk (PROSPER), in which all participants had either preexisting cardiovascular disease or were at risk of developing this condition.

\section{Patients and Methods}

\section{Study Design}

Data were obtained from PROSPER, a randomized, doubleblind, placebo-controlled trial designed to investigate the effect of pravastatin in prevention of vascular events in older individuals with pre-existing cardiovascular disease or risk factors thereof. This trial was conducted between 1997 and 2002 and included 5,804 men and women aged 70 to 82 years who were enrolled from 3 collaborating centers in Ireland, Scotland, and the Netherlands. Approximately $50 \%$ of the participants had cardiovascular disease including stable angina, intermittent claudication, stroke, TIA, myocardial infarction, and/or vascular surgery. The rest of the participants had 1 or more cardiovascu- lar risk factor, defined as hypertension, smoking, or diabetes mellitus. The primary outcome of the trial was the combined endpoint of definite or suspected death from coronary heart disease, nonfatal myocardial infarction, and fatal or nonfatal stroke during a mean follow-up period of 3.2 years. The institutional ethics committees of the 3 collaborating centers approved the study, and all participants gave written informed consent. $^{20,21}$

\section{Study Participants}

All participants had pre-existing cardiovascular disease or risk factors thereof (defined as a history of hypertension, diabetes mellitus, or current smoking). Participants with congestive heart failure, defined as New York Heart Association (NYHA) functional class III or IV, were excluded from the original PROSPER trial. ${ }^{20}$ No information on NYHA class I or II was available. For the present study, we additionally excluded participants with heart failure hospitalization during follow-up $(\mathrm{n}=205)$.

\section{NT-proBNP Measurements}

Blood samples were taken at 6 months after follow-up in ethylenediaminetetraacetic acid tubes. ${ }^{20}$ NT-proBNP was determined using electrochemiluminescence immunoassay on a Roche Modulator E170. A total of 394 participants had missing NTproBNP measurements. In line with existing literature on cutoff values in this age group, we defined 3 groups of NT-proBNP: low $(<100 \mathrm{ng} / \mathrm{l})$, middle (100-450 ng/l), and high NTproBNP $(\geq 450 \mathrm{ng} / \mathrm{l}) .{ }^{1}$ Furthermore, these cutoff values were chosen taking a pragmatic approach, to allow direct interpretation for clinical practice.

\section{Cognitive Function}

The MMSE was used to evaluate global cognitive function; participants with a baseline score $<24$ points were not included in PROSPER. Cognitive function was tested at baseline, at 9, 18, and 30 months, and at the end of the study. The time point of the measurement at the end of the study varied between 36 and 48 months; therefore, we performed the analysis with their individually varying time point, but report the results for the mean of these time points (at 42 months). Because PROSPER was conducted in 3 countries with in total 2 languages (Dutch and English), care was taken to select tests that are not sensitive to language. Furthermore, all analyses were adjusted for country. ${ }^{22}$ Four different neuropsychological tests were used to assess executive function, attention, and immediate and delayed memory. The Stroop Color and Word Test was used to test selective attention and reaction time. Participants were asked to read a color name, which was displayed in a color different from the color actually named. The outcome parameter was the total number of seconds to complete the test; a higher score indicates worse performance. General cognitive speed was tested by the Letter-Digit Coding Test. Participants had to match certain digits with letters according to a provided key. The outcome variable was the total number of correct entries in 60 seconds; therefore, higher scores represents better performance. The 
TABLE 1. Characteristics of Study Participants Grouped by NT-proBNP

\begin{tabular}{|c|c|c|c|c|}
\hline \multirow[b]{2}{*}{ Characteristic } & \multicolumn{3}{|c|}{ NT-proBNP } & \multirow[b]{2}{*}{$p^{a}$} \\
\hline & $\begin{array}{l}\text { Low, } \\
\mathrm{n}=1,818 \\
<100 \mathrm{ng} / 1\end{array}$ & $\begin{array}{l}\text { Middle, } \\
\mathrm{n}=2,698 \\
100-450 \mathrm{ng} / 1\end{array}$ & $\begin{array}{l}\text { High, } \\
\mathrm{n}=689 \\
\geq 450 \mathrm{ng} / 1\end{array}$ & \\
\hline \multicolumn{5}{|l|}{ Demographics } \\
\hline Age, yr, mean (SD) & $74.42(3.04)$ & $75.53(3.37)$ & $76.59(3.40)$ & $<0.001$ \\
\hline Female, No. [\%] & $850[46.8]$ & $1,490[55.2]$ & $360[52.2]$ & $<0.001$ \\
\hline $\begin{array}{l}\text { Education, age on leaving school, } \\
\text { yr, mean (SD) }\end{array}$ & $15.17(2.06)$ & $15.15(2.06)$ & $15.10(2.08)$ & 0.083 \\
\hline \multicolumn{5}{|l|}{ Vascular risk factors } \\
\hline History of hypertension, No. [\%] & $1,056[58.1]$ & $1,736[64.3]$ & $444[64.4]$ & $<0.001$ \\
\hline History of diabetes mellitus, No. [\%] & $245[13.5]$ & $245[9.1]$ & $57[8.3]$ & $<0.001$ \\
\hline History of stroke or TIA, No. [\%] & $189[10.4]$ & $301[11.2]$ & $85[12.3]$ & 0.371 \\
\hline History of myocardial infarction, No. [\%] & $120[6.6]$ & $369[13.7]$ & $177[25.7]$ & $<0.001$ \\
\hline History of vascular disease, No. [\%] & $630[34.7]$ & $1,246[46.2]$ & $393[57.0]$ & $<0.001$ \\
\hline Current smoker, No. [\%] & $536[29.5]$ & $667[24.7]$ & $175[25.4]$ & 0.001 \\
\hline Body mass index, $\mathrm{kg} / \mathrm{m}^{2}$, mean (SD) & $27.22(4.02)$ & $26.69(4.21)$ & $26.17(4.20)$ & $<0.001$ \\
\hline Total cholesterol, mmol/l, mean (SD) & $5.68(0.90)$ & $5.68(0.91)$ & $5.70(0.93)$ & 0.461 \\
\hline $\begin{array}{l}\text { Systolic blood pressure, } \mathrm{mmHg} \text {, } \\
\text { mean (SD) }\end{array}$ & $152.60(20.25)$ & $155.11(21.75)$ & $158.75(23.50)$ & $<0.001$ \\
\hline $\begin{array}{l}\text { Diastolic blood pressure, } \mathrm{mmHg} \text {, } \\
\text { mean (SD) }\end{array}$ & $84.02(10.95)$ & $83.73(11.33)$ & $83.38(12.01)$ & 0.158 \\
\hline Pulse pressure, mmHg, mean (SD) & $68.58(0.42)$ & $71.38(0.35)$ & $75.37(0.68)$ & $<0.001$ \\
\hline Mean arterial pressure, mmHg, mean (SD) & $106.88(0.30)$ & $107.53(0.25)$ & $108.51(0.49)$ & 0.001 \\
\hline $\begin{array}{l}\text { Systolic blood pressure trend, } \mathrm{mmHg} \text {, } \\
\text { mean }(\mathrm{SD})^{\mathrm{b}}\end{array}$ & $-0.97(7.94)$ & $-1.49(9.60)$ & $-2.88(12.44)$ & $<0.001$ \\
\hline $\begin{array}{l}\text { Diastolic blood pressure trend, } \mathrm{mmHg} \text {, } \\
\text { mean }(\mathrm{SD})^{\mathrm{b}}\end{array}$ & $-1.25(4.70)$ & $-1.52(5.25)$ & $-1.93(7.03)$ & 0.001 \\
\hline \multicolumn{5}{|l|}{$\begin{array}{l}\text { Blood pressure lowering medication, } \\
\text { No. [\%] }\end{array}$} \\
\hline Diuretics & $650[35.8]$ & $1,067[39.5]$ & $269[39.0]$ & $<0.001$ \\
\hline Loop & $153[8.4]$ & $327[12.1]$ & $107[15.5]$ & $<0.001$ \\
\hline Other & $497[27.3]$ & $740[27.4]$ & $162[23.5]$ & \\
\hline Calcium channel blockers & $459[25.2]$ & $692[25.6]$ & $151[21.9]$ & 0.125 \\
\hline Beta blockers & $241[13.3]$ & $831[30.8]$ & $273[39.6]$ & $<0.001$ \\
\hline ACE inhibitors & $279[15.3]$ & $421[15.6]$ & $134[19.4]$ & 0.031 \\
\hline eGFR, mean (SD) & $62.77(13.79)$ & $59.64(14.48)$ & $55.54(14.99)$ & $<0.001$ \\
\hline \multicolumn{5}{|c|}{$\begin{array}{l}{ }^{2} \text { Probability values were calculated using log-transformed NT-proBNP levels for continuous variables and chi-square tests for cate- } \\
\text { gorical variables. } \\
\text { EDefined as the regression coefficient per year. } \\
\text { ACE = angiotensin-converting enzyme; eGFR = estimated glomerular filtration rate; NT-proBNP }=\text { N-terminal pro-brain natri- } \\
\text { uretic peptide; SD = standard deviation; TIA = transient ischemic attack. }\end{array}$} \\
\hline
\end{tabular}


Picture-Word Learning Test was used to assess immediate and delayed memory. Fifteen pictures were presented, and participants were asked to recall as many pictures as possible in 3 trials. After 20 minutes, they were asked to repeat the pictures they remembered to measure their delayed recall. Outcome parameter was the accumulated number of correct recalled pictures, immediately and after 20 minutes. Higher scores thus indicate better performance. A detailed description of the cognitive tests has been published previously. ${ }^{22}$ Because treatment with pravastatin did not influence cognitive function during follow-up, we included participants from both pravastatin and placebo groups. $^{23}$

\section{Statistical Analysis}

Baseline characteristics of the study participants are reported as number (percentage) for categorical variables and mean (standard deviation) for continuous variables for each group of NTproBNP. Differences in categorical variables were tested by chisquare tests. Differences in continuous variables were tested with linear regression models. Because NT-proBNP levels were not normally distributed, we used log-transformed NT-proBNP levels to calculate $p$ values for continuous variables. To investigate the cross-sectional association of NT-proBNP with cognitive function, we used linear regression models. Logtransformed NT-proBNP levels were included as an independent variable; the outcome variable was the mean baseline score on each of the 4 cognitive function tests. Linear mixed models were used to examine the association between NT-proBNP and cognitive decline over time. The models included logtransformed NT-proBNP levels, time (in years), and the interaction term between time and log-transformed NT-proBNP levels.

We performed our analyses in 3 steps. In the first step, crude analyses were performed, in which we only adjusted for cognitive test version where appropriate. In the second step, we added the variables age, sex, education (age left school), country, and ApoE genotype to the model to investigate the potential influence of these factors on the associations (minimally adjusted model). Furthermore, in a fully adjusted model we also added the following potential confounders: cardiovascular diseases and risk factors at baseline (history of cerebrovascular and cardiovascular disease, hypertension, diabetes mellitus, smoking status, high-density lipoprotein and low-density lipoprotein cholesterol levels, triglycerides, systolic and diastolic blood pressure, body mass index), statin treatment, and estimated glomerular filtration rate (eGFR). Because the associations did not essentially change in various models, we present the results of the minimally and fully adjusted models only.

To further explore the influence of cardiovascular diseases and risk factors, additional analyses were performed in which we stratified for history of cardiovascular diseases and risk factors. To test whether the difference between participants with or without a history of cardiovascular disease or risk factor was significant, we calculated a $p$ value for interaction by using linear regression models. Furthermore, we performed additional sensitivity analyses in which we excluded (1) participants taking pravastatin treatment during follow-up; (2) participants with incident stroke and/or TIA; (3) participants with incident myocardial infarction; (4) participants with incident atrial fibrillation; (5) participants with vascular events, including coronary heart disease death, nonfatal myocardial infarction, nonfatal and fatal stroke, and/or TIA; (6) participants with NT-proBNP of $\geq 450 \mathrm{ng} / \mathrm{l}$; and (7) participants taking loop diuretics, beta blockers, or angiotensin-converting enzyme (ACE) inhibitors at baseline.

\section{Results}

Participants with heart failure hospitalization during follow-up were excluded $(n=205)$. A total of 394 participants had missing NT-proBNP measurements, resulting in a total number of 5,205 participants for the present study.

Table 1 shows characteristics of participants grouped by NT-proBNP levels. Participants with higher NT-proBNP levels were older and had a higher prevalence of hypertension, myocardial infarction, vascular disease, and smoking (all $p<0.001$ ). Body mass index was lower in participants with higher NT-proBNP levels ( $p$ value $<0.001)$. Systolic blood pressure, pulse pressure, and mean arterial blood pressure were higher among participants with higher NT-proBNP levels $(p<0.001$, $p<0.001$, and $p=0.001$, respectively). Furthermore, participants with higher NT-proBNP levels had a steeper decline in systolic and diastolic blood pressure during follow-up ( $p<0.001$ and $p=0.001$, respectively). Use of loop diuretics, beta blockers, and ACE inhibitors was higher in participants with higher NT-proBNP levels ( $p<0.001, p<0.001$, and $p=0.031$, respectively). Participants with higher NT-proBNP levels had lower eGFR $(p<0.001)$.

Table 2 shows the association of NT-proBNP levels with cognitive function at baseline. In the minimally adjusted model, participants with higher NT-proBNP levels had worse performance on the Stroop test $(p=0.003)$ and the Letter-Digit Coding Test $(p<0.001)$. The same trend was observed for immediate and delayed Picture-Word Learning Test, showing that participants with higher NT-proBNP levels had worse performance, albeit these associations were not significant ( $p=0.060$ and $p=0.066$, respectively). When further adjusting for prevalent cardiovascular diseases or risk factors at baseline, the estimates of the difference in cognitive function between the groups remained essentially the same. The association of NT-proBNP levels with the Stroop test and Letter-Digit Coding Test in the fully adjusted model remained significant $(p=0.003$ and $p<0.001$, respectively), whereas for immediate and delayed Picture-Word Learning Test the associations were 
TABLE 2. Association of NT-proBNP with Baseline Cognitive Function

\begin{tabular}{|c|c|c|c|c|}
\hline \multirow[b]{2}{*}{ Cognitive Tests } & \multicolumn{3}{|c|}{ NT-proBNP } & \multirow[b]{2}{*}{$p^{\mathbf{a}}$} \\
\hline & $\begin{array}{l}\text { Low, } n=1,818, \\
<100 \mathrm{ng} / 1\end{array}$ & $\begin{array}{l}\text { Middle, } \mathrm{n}=2,698 \text {, } \\
100-450 \mathrm{ng} / 1\end{array}$ & $\begin{array}{l}\text { High, } n=689 \\
\geq 450 \mathrm{ng} / 1\end{array}$ & \\
\hline \multicolumn{5}{|l|}{ Stroop, seconds } \\
\hline Minimally adjusted model & $64.37(1.46)$ & $64.15(1.42)$ & $67.40(1.63)$ & 0.003 \\
\hline Fully adjusted model & $66.23(1.56)$ & $66.09(1.53)$ & $69.30(1.72)$ & 0.003 \\
\hline \multicolumn{5}{|l|}{ LDCT, digits coded } \\
\hline Minimally adjusted model & $23.94(0.41)$ & $23.54(0.40)$ & $23.02(0.46)$ & $<0.001$ \\
\hline Fully adjusted model & $23.33(0.44)$ & $22.88(0.43)$ & $22.31(0.48)$ & $<0.001$ \\
\hline \multicolumn{5}{|l|}{ PLTi, pictures remembered } \\
\hline Minimally adjusted model & $9.58(0.11)$ & $9.52(0.11)$ & $9.44(0.12)$ & 0.060 \\
\hline Fully adjusted model & $9.49(0.12)$ & $9.44(0.12)$ & $9.37(0.13)$ & 0.091 \\
\hline \multicolumn{5}{|l|}{ PLTd, pictures remembered } \\
\hline Minimally adjusted model & $10.43(0.16)$ & $10.40(0.15)$ & $10.29(0.17)$ & 0.066 \\
\hline Fully adjusted model & $10.22(0.17)$ & $10.19(0.16)$ & $10.08(0.18)$ & 0.062 \\
\hline \multicolumn{5}{|c|}{$\begin{array}{l}\text { Data represent mean (standard error) score of each cognitive function test. } \\
\text { Minimally adjusted model: adjusted for age, sex, country, education (age on leaving school), ApoE genotype, treatment group, and } \\
\text { test version for LDCT and PLT. } \\
\text { Fully adjusted model: minimally adjusted model + adjustments for history of cerebrovascular and cardiovascular disease, history of } \\
\text { hypertension, history of diabetes mellitus, current smoking, baseline systolic and diastolic blood pressure, high-density lipoprotein } \\
\text { and low-density lipoprotein cholesterol, triglycerides, body mass index, and estimated glomerular filtration rate. } \\
\text { aProbability values were calculated using the continuous value of log-transformed NT-proBNP levels. } \\
\text { LDCT = Letter-Digit Coding Test; NT-proBNP = N-terminal pro-brain natriuretic peptide; PLTd = Picture-Word Learning Test, } \\
\text { delayed; PLTi = Picture-Word Learning Test, immediate. }\end{array}$} \\
\hline
\end{tabular}

not significant $(p=0.091$ and $p=0.062$, respectively). Data on the association of NT-proBNP with cognitive function from crude models did not materially differ from minimally and fully adjusted models (data not shown).

Table 3 and Figure 1 show the association of NTproBNP levels with changes in cognitive function during a mean follow-up period of 3.2 years. Participants with higher NT-proBNP levels had a steeper cognitive decline on the Stroop test, Letter-Digit Coding Test, and immediate and delayed Picture-Word Learning Test (all $p \leq 0.001$ ). Again, further adjustments for prevalent cardiovascular diseases or risk factors at baseline did not appreciably alter the observed associations (all $p \leq 0.001$ ). The association of NT-proBNP levels with cognitive decline from crude models did not materially differ from adjusted models (data not shown).

To further explore the influence of cardiovascular diseases and risk factors, we performed additional analyses in which we stratified for history of various cardiovascular diseases and risk factors, and tested for interaction. Figure 2 shows the association of NT-proBNP levels with cognitive decline, stratified by history of cardiovascular diseases and risk factors. There was no significant difference in change in cognitive function during follow-up between participants with and without cardiovascular diseases or risk factors, except on the Letter-Digit Coding Test for participants with a history of stroke and/or TIA and myocardial infarction. Participants with previous stroke and/or TIA had a less steep decline on the LetterDigit Coding Test $(p$ for interaction $=0.003$ ), whereas participants with previous myocardial infarction had a steeper decline on the Letter-Digit Coding Test ( $p$ for interaction $=0.008)$. However, no such differences were observed for participants with previous stroke and/or TIA or myocardial infarction on any of the other cognitive tests.

Furthermore, we performed additional sensitivity analyses to investigate whether the association between NT-proBNP levels and cognitive function and decline could be affected by (1) participants taking pravastatin treatment during follow-up ( $\mathrm{n}=2,588)$; (2) participants with incident stroke and/or TIA during follow-up ( $\mathrm{n}=355)$; (3) participants with incident myocardial 
TABLE 3. Association of NT-proBNP with Cognitive Decline during Follow-up

\begin{tabular}{|c|c|c|c|c|}
\hline \multirow[b]{2}{*}{ Cognitive Tests } & \multicolumn{3}{|c|}{ NT-proBNP } & \multirow[b]{2}{*}{$p^{a}$} \\
\hline & $\begin{array}{l}\text { Low, } n=1,818, \\
<100 \mathrm{ng} / 1\end{array}$ & $\begin{array}{l}\text { Middle, } n=2,698 \text {, } \\
100-450 \mathrm{ng} / 1\end{array}$ & $\begin{array}{l}\text { High, } n=689 \\
\geq 450 \mathrm{ng} / 1\end{array}$ & \\
\hline \multicolumn{5}{|l|}{ Stroop, seconds } \\
\hline Minimally adjusted model & $0.46(0.11)$ & $0.71(0.09)$ & $1.04(0.26)$ & 0.001 \\
\hline Fully adjusted model & $0.47(0.11)$ & $0.72(0.09)$ & $1.04(0.26)$ & 0.001 \\
\hline \multicolumn{5}{|l|}{ LDCT, digits coded } \\
\hline Minimally adjusted model & $-0.32(0.02)$ & $-0.36(0.02)$ & $-0.46(0.04)$ & 0.001 \\
\hline Fully adjusted model & $-0.32(0.02)$ & $-0.35(0.02)$ & $-0.47(0.04)$ & $<0.001$ \\
\hline \multicolumn{5}{|l|}{ PLTi, pictures remembered } \\
\hline Minimally adjusted model & $-0.00(0.01)$ & $-0.03(0.01)$ & $-0.05(0.02)$ & $<0.001$ \\
\hline Fully adjusted model & $0.00(0.01)$ & $-0.02(0.01)$ & $-0.04(0.02)$ & $<0.001$ \\
\hline \multicolumn{5}{|l|}{ PLTd, pictures remembered } \\
\hline Minimally adjusted model & $-0.05(0.01)$ & $-0.06(0.01)$ & $-0.10(0.03)$ & 0.001 \\
\hline Fully adjusted model & $-0.03(0.01)$ & $-0.05(0.01)$ & $-0.10(0.03)$ & 0.001 \\
\hline \multicolumn{5}{|c|}{ 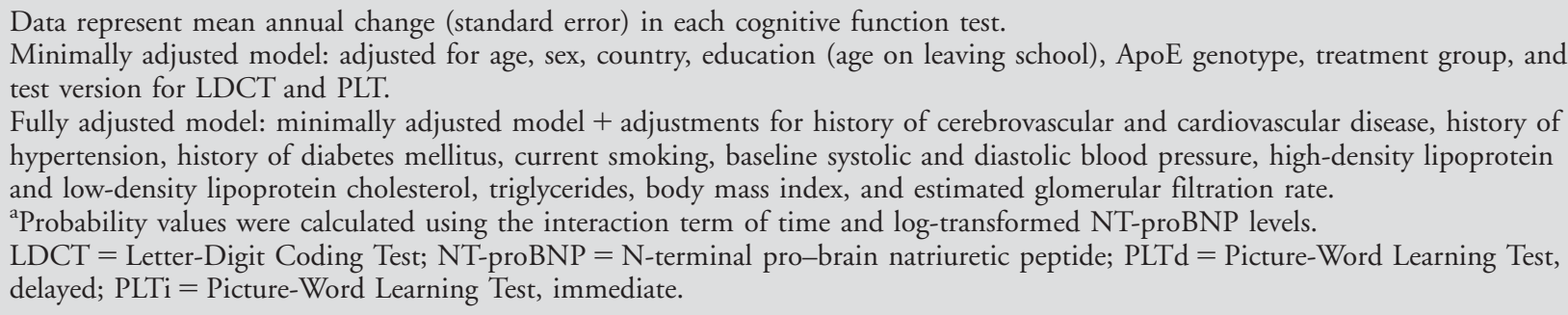 } \\
\hline
\end{tabular}

infarction during follow-up $(\mathrm{n}=339)$; (4) participants with incident atrial fibrillation during follow-up ( $\mathrm{n}=421)$; (5) participants with vascular events, including coronary heart disease death, nonfatal myocardial infarction, nonfatal and fatal stroke, and/or TIA during follow-up ( $\mathrm{n}=648)$; (6) participants with NT-proBNP levels of $\geq 450 \mathrm{ng} / \mathrm{l}$; and (7) participants taking loop diuretics $(n=588)$, beta blockers $(n=1,345)$, or ACE inhibitors $(n=834)$ at baseline. Exclusion of these participants did not essentially change our results (data available on request).

\section{Discussion}

In this prospective cohort study including $>5,000$ men and women with a mean age of 75 years, we showed that participants with higher NT-proBNP levels had worse cognitive function and steeper cognitive decline during a mean period of 3.2 years. These associations were independent of cardiovascular diseases and risk factors.

Our findings are in line with previous crosssectional studies showing that higher NT-proBNP levels were associated with worse memory and with lower global and executive cognitive function. ${ }^{1-4}$ Only a few longitudinal studies with a limited number of participants investigated the association between NT-proBNP and cognition during follow-up. They showed that higher NT-proBNP levels are associated with a steeper decline in MMSE scores and a higher incidence of dementia during a mean follow-up period of 5 years. ${ }^{5,6}$ To our knowledge, this is the first study reporting on the association of NT-proBNP and cognitive function and decline, using an extended standardized test battery over a mean follow-up period of 3.2 years, in a large cohort of older adults with pre-existing cardiovascular disease or risk factors therefor.

Brain natriuretic peptide (BNP) and the biologically inactive $\mathrm{N}$-terminal proBNP are secreted by the ventricles of the heart in response to excessive stretching of cardiomyocytes. ${ }^{24}$ BNP has favorable physiological properties, including increased natriuresis and diuresis, relaxation of vascular smooth muscle cells, and inhibition of the renin-angiotensin-aldosterone axis, eventually causing a reduction in blood pressure and ventricular 

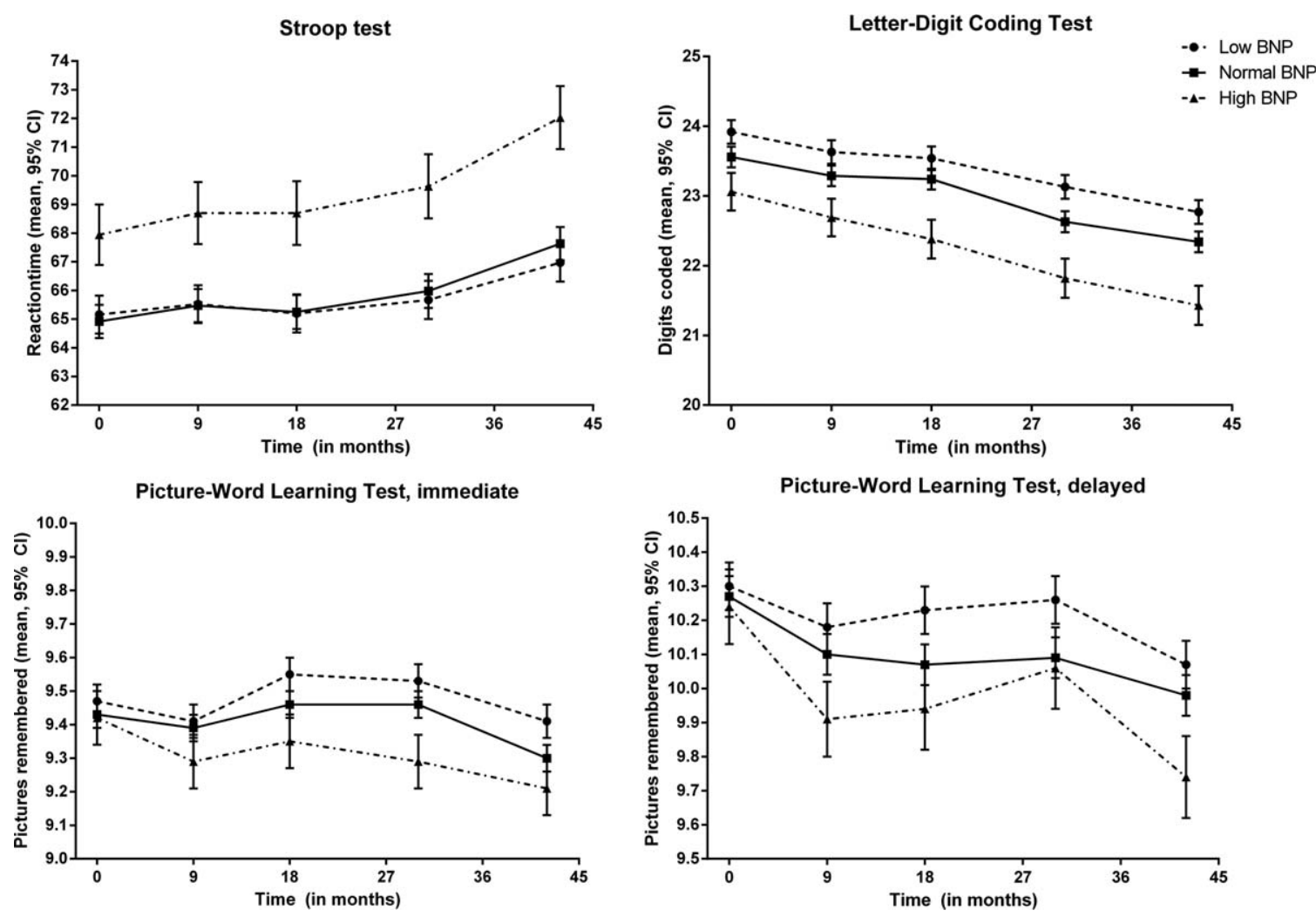

FIGURE 1: Association of N-terminal pro-brain natriuretic peptide (NT-proBNP) with cognitive decline during follow-up. Data represent mean score (95\% confidence interval) of each cognitive test during follow-up, in each group of NT-proBNP. Because the time point of the measurement at the end of the study varied between 36 and 48 months, the mean of these time points (42 months) is reported. Probability values were calculated using the interaction term of time and log-transformed NT-proBNP levels. Adjustments were made for age, sex, country, education, ApoE genotype, treatment group, and test version where appropriate. $\mathrm{Cl}$, confidence interval.

preload. ${ }^{24}$ Our results showed that higher NT-proBNP levels were associated with higher systolic blood pressure. Initially, higher systolic blood pressure might increase the ventricular stress of cardiomyocytes and therefore cause an increased release of NT-proBNP. In case of chronic ventricular stress, this might further proceed to reduced cardiac function and heart failure. Subsequently, cardiac output will be reduced and blood pressure will be lower.

Different explanations can be proposed for the observed association of NT-proBNP with cognitive decline. First, NT-proBNP and cognitive decline are highly likely to reflect underlying cardiovascular damage and therefore stem from common causes. Previous studies have shown that NT-proBNP levels have a prognostic value for the occurrence of cardiovascular events, such as myocardial infarction, atrial fibrillation, coronary heart disease, unstable angina, stroke, and TIA. ${ }^{12,15,25,26}$ This has also been demonstrated in subjects with elevated NTproBNP levels, but without clinical heart failure. ${ }^{27}$ Furthermore, NT-proBNP levels provide predictive information for use in risk stratification in nonfatal cardiac events, stroke, and mortality in a range of populations including patients with diabetes. ${ }^{26,28-30}$ These cardiovas- cular and metabolic diseases are closely linked to cognitive dysfunction and dementia. ${ }^{18,19}$ This is in line with the finding that high NT-proBNP levels are associated with an increased prevalence of cardiovascular diseases and risk factors in the population under study. However, when adjusting and stratifying our analyses for cardiovascular diseases and risk factors, our results did not appreciably alter. Furthermore, excluding participants with incident myocardial infarction, stroke, and/or TIA showed the same results. Nevertheless, we cannot rule out the possibility that unmeasured cardiovascular risk factors resulted in both increased NT-proBNP and cognitive decline. Second, impaired cardiac function may activate the renin-angiotensin system, which in turn has been associated with cognitive decline. ${ }^{31}$ In line with this evidence, observational studies have suggested that subjects receiving angiotensin receptor blockers may have a lower risk of developing dementia. ${ }^{32,33}$ Because only a small number of participants used angiotensin receptor blockers in the population under study $(\mathrm{n}<100)$, we could not further investigate this issue. Third, because natriuretic peptides have first been identified in porcine brain extract, one could hypothesize that NT-proBNP 


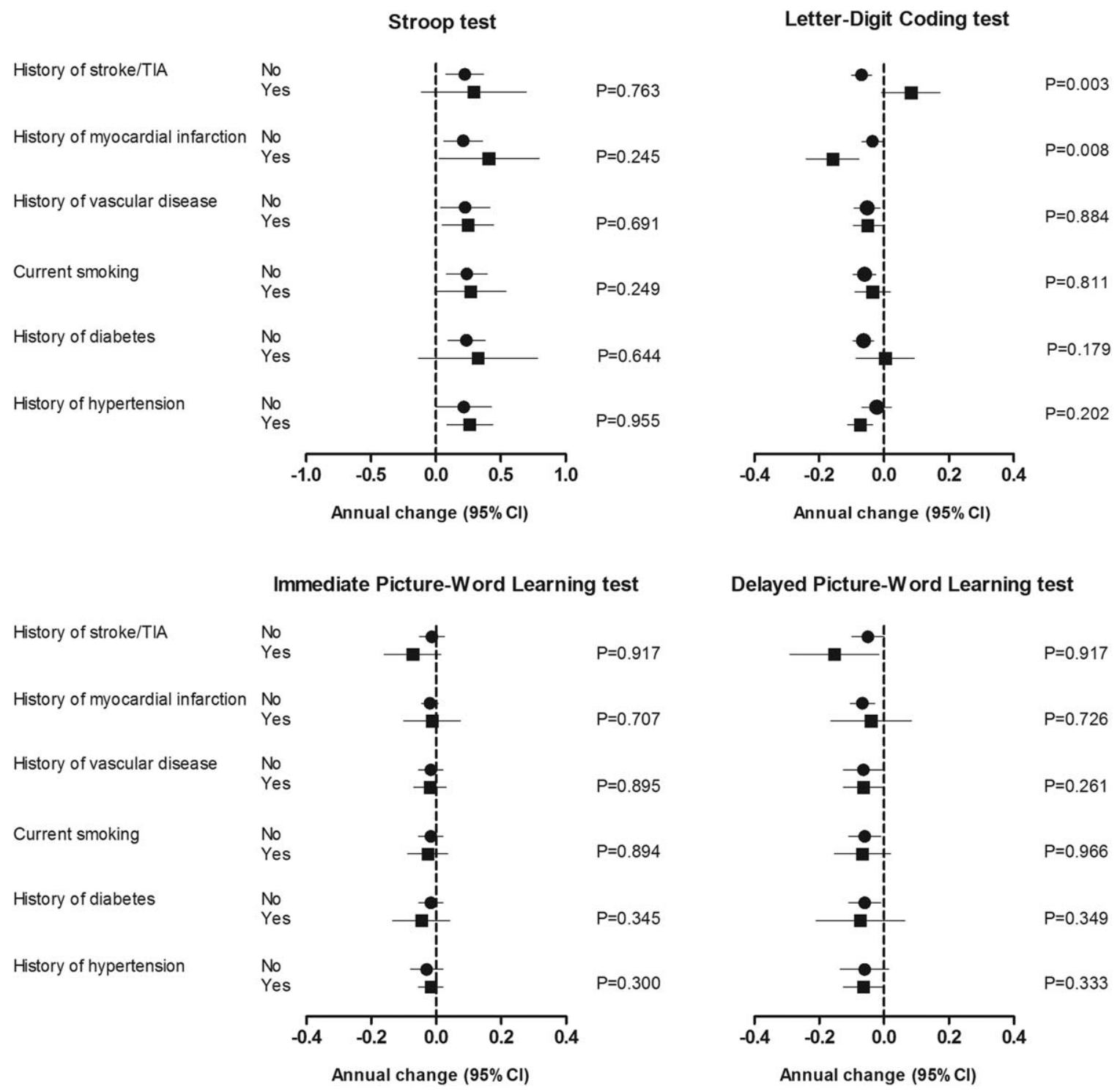

FIGURE 2: Association of N-terminal pro-brain natriuretic peptide (NT-proBNP) with cognitive decline during follow-up, stratified by cardiovascular diseases and risk factors. Data represent mean annual change ( $95 \%$ confidence interval [CI]) per $1 \mathrm{ng} / \mathrm{l}$ increase in log-transformed NT-proBNP for each cognitive test, stratified by cardiovascular diseases. Probability values were calculated using the interaction term of cardiovascular disease and log-transformed NT-proBNP level and represent the statistical difference in annual change in cognitive function between participants with and without cardiovascular disease or risk factors. Adjustments were made for age, sex, country, education, ApoE genotype, treatment group, and test version where appropriate. $\mathrm{TIA}=$ transient ischemic attack.

could have a direct effect in the brain. Although there is evidence that natriuretic peptides have receptors on endothelial cells, it is to our knowledge unknown whether NT-proBNP alters cerebral autoregulation. ${ }^{7,34}$ A fourth explanation might be that high NT-proBNP levels in subjects without advanced stages of heart failure indicate suboptimal left ventricular functioning with subsequent decreased cardiac output and cerebral hypoperfusion. ${ }^{9,16}$ Cerebral hypoperfusion, which impairs the delivery of oxygen and nutrients to the brain, has been associated with cognitive dysfunction and dementia. ${ }^{7-9}$ Although this explanation seems plausible, there is a need for interventional studies investigating the influence of improvement in cardiac function with its subsequent influence on cerebral perfusion, and eventually the prevention of cognitive decline in old age.

The present study found that participants with previous stroke and/or TIA had a less steep decline on the Letter-Digit Coding Test, which is unexpected and not in line with previous literature. Nevertheless, no differences were observed for participants with previous stroke and/or TIA on the Stroop test, immediate Picture-Word Learning Test, and delayed Picture Word Learning Test. Furthermore, as there was no significant association between NT-proBNP levels and history of stroke and/or TIA, we could not explain this association from a 
biologically perspective. Therefore, we believe that the most likely explanation for this finding is chance.

Major strengths of this study include the large sample size of $>5,000$ older participants and the repeated use of an extended standardized cognitive test battery to assess cognitive function over a mean follow-up period of 3.2 years. Furthermore, in contrast to previous studies, participants with NYHA functional class III/IV were excluded, which gave us the opportunity to investigate NT-proBNP in relation to cognitive function and decline in participants without advanced stages of clinical heart failure. However, a limitation of the study is that there was no information on the incidence of dementia during follow-up, nor was there information on cardiac functioning or NYHA class I or II. We might therefore have included participants with (beginning stages of) clinical heart failure, without ever being diagnosed with this condition. However, excluding participants with NT-proBNP levels of $\geq 450 \mathrm{ng} / \mathrm{l}$ showed essentially the same results. As high NT-proBNP levels have been recognized as a predictor of heart failure, this finding further suggests an association between NTproBNP and cognitive decline. ${ }^{35}$ Furthermore, our study population consisted of older participants at risk of cardiovascular diseases with relatively preserved cognitive function (MMSE $\geq 24$ points), which might limit extrapolation of our findings to a general population of older subjects.

In conclusion, higher NT-proBNP levels associate with worse cognitive function and steeper cognitive decline in older adults, independent of cardiovascular diseases and risks. Further studies to unravel the underlying mechanisms are warranted.

\section{Acknowledgment}

The original PROSPER clinical trial was funded by an investigator-initiated grant from Bristol-Myers Squibb, USA. NT-proBNP measurements were funded by a grant from Biobanking and Biomolecular Research Infrastructure The Netherlands (grant number CP2011-33). Both companies had no involvement in the design and conduct of the study; collection, management, analysis, and interpretation of the data; preparation, review, and approval of the manuscript; or decision to submit the manuscript for publication.

\section{Potential Conflicts of Interest}

Nothing to report.

\section{References}

1. Daniels LB, Laughlin GA, Kritz-Silverstein D, et al. Elevated natriuretic peptide levels and cognitive function in community-dwelling older adults. Am J Med 2011;124:670-678.
2. Feinkohl I, Sattar N, Welsh P, et al. Association of N-terminal probrain natriuretic peptide with cognitive function and depression in elderly people with type 2 diabetes. PLoS One 2012;7:e44569.

3. Gunstad J, Poppas A, Smeal S, et al. Relation of brain natriuretic peptide levels to cognitive dysfunction in adults $>55$ years of age with cardiovascular disease. Am J Cardiol 2006:98:538-540.

4. van den Hurk K, Reijmer YD, van den Berg E, et al. Heart failure and cognitive function in the general population: the Hoorn Study. Eur J Heart Fail 2011;13:1362-1369.

5. Feinkohl I, Keller M, Robertson CM, et al. Clinical and subclinical macrovascular disease as predictors of cognitive decline in older patients with type 2 diabetes: the Edinburgh Type 2 Diabetes Study. Diabetes Care 2013;36:2779-2786.

6. Kerola T, Nieminen T, Hartikainen S, et al. B-Type natriuretic peptide as a predictor of declining cognitive function and dementiaa cohort study of an elderly general population with a 5-year follow-up. Ann Med 2010;42:207-215.

7. Georgiadis D, Sievert M, Cencetti S, et al. Cerebrovascular reactivity is impaired in patients with cardiac failure. Eur Heart J 2000; 21:407-413.

8. Zuccala G, Cattel C, Manes-Gravina E, et al. Left ventricular dysfunction: a clue to cognitive impairment in older patients with heart failure. J Neurol Neurosurg Psychiatry 1997;63:509-512.

9. Zuccala G, Onder G, Pedone C, et al. Hypotension and cognitive impairment: selective association in patients with heart failure. Neurology 2001;57:1986-1992.

10. Bornstein RA, Starling RC, Myerowitz PD, Haas GJ. Neuropsychological function in patients with end-stage heart failure before and after cardiac transplantation. Acta Neurol Scand 1995;91:260-265.

11. Grimm M, Yeganehfar W, Laufer G, et al. Cyclosporine may affect improvement of cognitive brain function after successful cardiac transplantation. Circulation 1996;94:1339-1345.

12. Kistorp C, Raymond I, Pedersen F, et al. N-terminal pro-brain natriuretic peptide, $\mathrm{C}$-reactive protein, and urinary albumin levels as predictors of mortality and cardiovascular events in older adults. JAMA 2005:293:1609-1616.

13. Pedersen F, Raymond I, Kistorp C, et al. N-terminal pro-brain natriuretic peptide in arterial hypertension: a valuable prognostic marker of cardiovascular events. J Card Fail 2005;11(5 suppl):S70-S75.

14. Vaes B, de Ruijter W, Degryse J, et al. Clinical relevance of a raised plasma $\mathrm{N}$-terminal pro-brain natriuretic peptide level in a population-based cohort of nonagenarians. J Am Geriatr Soc 2009;57:823-829.

15. Wang TJ, Larson MG, Levy $D$, et al. Plasma natriuretic peptide levels and the risk of cardiovascular events and death. $N$ Engl $J$ Med 2004;350:655-663.

16. Mishra RK, Li Y, Ricardo AC, et al. Association of N-terminal proB-type natriuretic peptide with left ventricular structure and function in chronic kidney disease (from the Chronic Renal Insufficiency Cohort [CRIC]). Am J Cardiol 2013;111:432-438.

17. Uraizee I, Cheng $\mathrm{S}$, Hung $\mathrm{CL}$, et al. Relation of $\mathrm{N}$-terminal pro-Btype natriuretic peptide with diastolic function in hypertensive heart disease. Am J Hypertens 2013;26:1234-1241.

18. Gorelick PB, Scuteri A, Black SE, et al. Vascular contributions to cognitive impairment and dementia: a statement for healthcare professionals from the American Heart Association/American Stroke Association. Stroke 2011;42:2672-2713.

19. Pantoni L. Cerebral small vessel disease: from pathogenesis and clinical characteristics to therapeutic challenges. Lancet Neurol 2010:9:689-701.

20. Shepherd J, Blauw GJ, Murphy MB, et al. The design of a prospective study of Pravastatin in the Elderly at Risk (PROSPER) PROSPER Study Group. PROspective Study of Pravastatin in the Elderly at Risk. Am J Cardiol 1999;84:1192-1197. 
21. Shepherd J, Blauw GJ, Murphy MB, et al. Pravastatin in elderly individuals at risk of vascular disease (PROSPER): a randomised controlled trial. Lancet 2002;360:1623-1630.

22. Houx PJ, Shepherd J, Blauw GJ, et al. Testing cognitive function in elderly populations: the PROSPER study. PROspective Study of Pravastatin in the Elderly at Risk. J Neurol Neurosurg Psychiatry 2002;73:385-389

23. Trompet $\mathrm{S}$, van Vliet $\mathrm{P}$, de Craen AJ, et al. Pravastatin and cognitive function in the elderly. Results of the PROSPER study. J Neurol 2010;257:85-90

24. de Lemos JA, McGuire DK, Drazner MH. B-Type natriuretic peptide in cardiovascular disease. Lancet 2003;362:316-322.

25. Hijazi Z, Oldgren J, Andersson U, et al. Cardiac biomarkers are associated with an increased risk of stroke and death in patients with atrial fibrillation: a randomized evaluation of long-term anticoagulation therapy (RE-LY) substudy. Circulation 2012;125:1605-1616.

26. Hijazi Z, Wallentin L, Siegbahn A, et al. N-terminal pro-B-type natriuretic peptide for risk assessment in patients with atrial fibrillation: insights from the ARISTOTLE Trial (Apixaban for the Prevention of Stroke in Subjects With Atrial Fibrillation). J Am Coll Cardiol 2013;61:2274-2284.

27. Di $A E$, Chowdhury R, Sarwar N, et al. B-type natriuretic peptides and cardiovascular risk: systematic review and meta-analysis of 40 prospective studies. Circulation 2009;120:2177-2187.

28. de Lemos JA, Morrow DA, Gibson CM, et al. The prognostic value of serum myoglobin in patients with non-ST-segment eleva- tion acute coronary syndromes. Results from the TIMI 11B and TACTICS-TIMI 18 studies. J Am Coll Cardiol 2002;40:238-244.

29. Hillis GS, Welsh P, Chalmers J, et al. The relative and combined ability of high-sensitivity cardiac troponin $\mathrm{T}$ and $\mathrm{N}$-terminal pro-Btype natriuretic peptide to predict cardiovascular events and death in patients with type 2 diabetes. Diabetes Care 2014;37: 295-303.

30. Welsh $\mathrm{P}$, Doolin $\mathrm{O}$, Willeit $\mathrm{P}$, et al. N-terminal pro-B-type natriuretic peptide and the prediction of primary cardiovascula events: results from 15-year follow-up of WOSCOPS. Eur Heart $J$ 2013;34:443-450.

31. Unger $T$. The role of the renin-angiotensin system in the development of cardiovascular disease. Am J Cardiol 2002;89:3A-9A.

32. Li NC, Lee A, Whitmer RA, et al. Use of angiotensin receptor blockers and risk of dementia in a predominantly male population: prospective cohort analysis. BMJ 2010;340:b5465.

33. Muller $M$, van der Graaf $Y$, Visseren $F L$, et al. Hypertension and longitudinal changes in cerebral blood flow: the SMART-MR study. Ann Neurol 2012;71:825-833.

34. Kuhn M. Endothelial actions of atrial and B-type natriuretic peptides. Br J Pharmacol 2012;166:522-531.

35. Wannamethee $\mathrm{SG}$, Welsh $\mathrm{P}$, Whincup $\mathrm{PH}$, et al. N-terminal pro brain natriuretic peptide but not copeptin improves prediction of heart failure over other routine clinical risk parameters in older men with and without cardiovascular disease: population-based study. Eur J Heart Fail 2014;16:25-32. 